\title{
Tumour sidedness and intrinsic subtypes in patients with stage II/III colon cancer: analysis of NSABP C-07 (NRG Oncology)
}

S Rim Kim ${ }^{1}$, Nan Song ${ }^{1}$, Greg Yothers ${ }^{1,2}$, Patrick G Gavin ${ }^{1}$, Carmen J Allegra ${ }^{1,3}$, Soonmyung Paik ${ }^{1,4}$ and Katherine L Pogue-Geile*,1

${ }^{1}$ National Surgical Adjuvant Breast and Bowel Project/NRG Oncology, Pittsburgh, PA 15213, USA; ${ }^{2}$ University of Pittsburgh, Department of Biostatistics, Pittsburgh, PA 15213, USA; ${ }^{3}$ Department of Medicine, University of Florida Health, Gainesville, FL 32610, USA and ${ }^{4}$ Severance Biomedical Science Institute Division of Medical Oncology and Yonsei University College of Medicine, Seoul 03722, Republic of Korea

Background: We tested the association of colon tumour sidedness with prognosis and with molecular subtypes recently shown to be predictive of oxaliplatin benefit in stage III colon cancer.

Methods: NSABP/NRG C-07 trial ( $N=1603)$ was used to determine association of tumour sidedness with molecular subtypes and recurrence-free survival (RFS) and overall survival (OS).

Results: Sidedness was associated with molecular subtypes except stem-like/CMS4 subtype. Patients with stage III, left-sided tumours showed superior OS but not RFS. Sidedness was not associated with prediction of oxaliplatin benefit when combined with 5-Fu + LV. However, greater benefit from oxaliplatin was observed in a small subset of stage III patients with left-sided, enterocyte-subtype tumours (interaction $\mathrm{HR}=0.17, P=0.01$ ).

Conclusions: Sidedness was associated with molecular subtypes and was predictive of OS in stage III colon cancer but was not predictive of RFS or oxaliplatin benefit in C-07. Molecular subtypes may provide more predictive value for oxaliplatin benefit than tumour sidedness.

Several recent studies have indicated that primary tumour sidedness may be prognostic and predictive of anti-EGFR therapy response in metastatic colorectal cancer. The ad hoc analysis of CALGB/SWOG 80405, a trial of cetuximab or bevacizumab combined with FOLFOX or FOLFIRI, showed better overall patient survival with KRAS wild-type metastatic colorectal cancer when the tumour was left-sided, regardless of treatment subgroup (Venook et al, 2016). Similar trials, including CRYSTAL and FIRE3 , reported that patients with left-sided tumours had a better prognosis and also received greater benefit from cetuximab added to first- or second-line chemotherapy than did patients with rightsided tumours (Tejpar et al, 2016).

In a population-based study of stage III and IV colon cancer, patients with right-sided tumours had inferior survival in the adjuvant setting (Schrag et al, 2016). Results seen in the analysis of patients enrolled into adjuvant clinical trials VICTOR and QUASAR2 $(N=1935)$ were consistent with those findings. Cancer in the right-sided colon was associated with poorer prognosis for overall survival (OS) but not for recurrence-free survival (RFS), which was equivalent for left- and right-sided tumours (Kerr et al, 2016). This discrepancy between OS and RFS is a result of the poor survival after recurrence (SAR) in patients with right-sided tumours compared with patients with left-sided tumours. Similar observations were made when BRAF mutations were assessed for prognosis; no association was seen when time to recurrence was used, but BRAF was associated with poor prognosis if OS or SAR were used as end points (Gavin et al, 2012). The association of right-sided tumours with poor prognosis was maintained even

*Correspondence: KL Pogue-Geile; E-mail: katherine.pogue-geile@nsabp.org

Received 23 May 2017; revised 14 November 2017; accepted 15 November 2017; published online 14 December 2017

(C) 2018 Cancer Research UK. All rights reserved 0007-0920/18 
when the models were adjusted for many clinical and molecular variables, including MSI, CIN, KRAS, and BRAF status, suggesting that BRAF and MSI status, which are known to be more common on the right side and to influence outcomes, are not the sole drivers responsible for the poor prognosis of right-sided tumours.

A series of gene expression signatures recently developed to classify colorectal cancer (De Sousa E Melo et al, 2013; Sadanandam et al, 2013; Guinney et al, 2015) revealed that each subtype had distinct molecular features and prognoses. When the predictive values of these signatures were retrospectively tested for oxaliplatin benefit in the NSABP/NRG C-07 trial, the Colorectal Cancer Assigner (CRCA) specifically identified a statistically significant interaction between enterocyte subtype and oxaliplatin benefit in the discovery cohort and a trend for significance in the validation cohort (Song et al, 2016). Notably, the association of poor prognosis in patients with the stem-like subtype was robustly identified regardless of subtype classifications and stages.

In this study, we examined the association of primary tumour location with prognosis and prediction for oxaliplatin benefit in C-07 and in subsets of C-07 based on molecular subtypes. We chose to focus our analyses using CRCA subtyping because our evidence suggested that it more clearly defined an oxaliplatin benefit group than did Consensus Molecular Subtype (CMS) subtyping (Song et al, 2016). The CMS2 subtype received benefit from oxaliplatin, but the degree of benefit and significance was greater in the CRCA enterocyte subtype. Subsetting the CMS2 subtype into enterocyteand TA-like showed that oxaliplatin benefit was limited to the CMS2-enteroctye sub-subtype.

\section{MATERIALS AND METHODS}

The NSABP C-07 trial was approved by local human investigation committees or institutional review boards in accordance with assurances filed with and approved by the Department of Health and Human Services. Written informed consent was required for participation.

NCounter data for all properly consented patients from NSABP/ NRG C-07 were included $(N=1603)$ in this ad hoc analysis. Subtyping methods based on gene expression profiling have been described previously (Song et al, 2016). We defined sidedness of tumours as did other previous studies (Tejpar et al, 2016; Venook et al, 2016). Ten cases with mixed combination of right- and leftsided tumours were excluded for the analysis. Associations of clinical variables and mutations with sidedness were analysed by means of the $\chi^{2}$ test. The primary end point in this study is time to recurrence (time from random assignment to recurrence censor for death (competing risk) or last follow-up). For each variable, we assessed (1) prognostic significance using univariate Cox models and (2) predictive values for oxaliplatin benefit using Cox models with an interaction term between treatment and tested variable.

\section{RESULTS}

Of the 1603 cases, 719 (44.9\%) were left sided, 874 (54.5\%) were right sided, and 10 had multiple locations on both sides simultaneously; the latter were excluded from further analysis. Stage; grade; obstruction; and BRAF, KRAS, and PIK3CA mutations are strongly associated with sidedness. Right- and leftsided tumours also showed different distributions of intrinsic subtypes regardless of molecular classifiers $(P<0.001)$, with the exception of the CRCA (Colorectal Cancer Assigner) stem-like subtype and the CMS4 subtype (Supplementary Table 1). Gobletlike, inflammatory, CMS1 (immune), and CMS3 (metabolic) were predominant in the right colon, whereas enterocyte, transitamplifying (TA), and CMS2 (canonical) were more frequent in the left (overall $P<0.001$; Supplementary Table 1; Figure 1). Stem-like/ CMS4 (mesenchymal) subtypes defined similar subsets of patients with poor prognosis and have similar distribution in right- and left- sided tumours.

Sidedness was not associated with prognosis in the entire C-07 cohort (Figure 2A and B) and not in stage III patients based on RFS (HR: 0.86 (CI: 0.71-1.04), $P=0.12$ ), but sidedness was associated with prognosis based on OS (0.78 (CI: 0.65-0.95) $P=0.011$; Figure 2C and D).

Sidedness was not associated with prediction of stage III patients who received greater benefit from oxaliplatin combined with 5$\mathrm{FU}+$ leucovorin chemotherapy (RFS-interaction $\mathrm{HR}=0.94$, $P=0.78 ; \quad$ Figure $3 \mathrm{~A} ; \quad$ OS-interaction $\quad \mathrm{HR}=0.85, \quad P=0.42$;
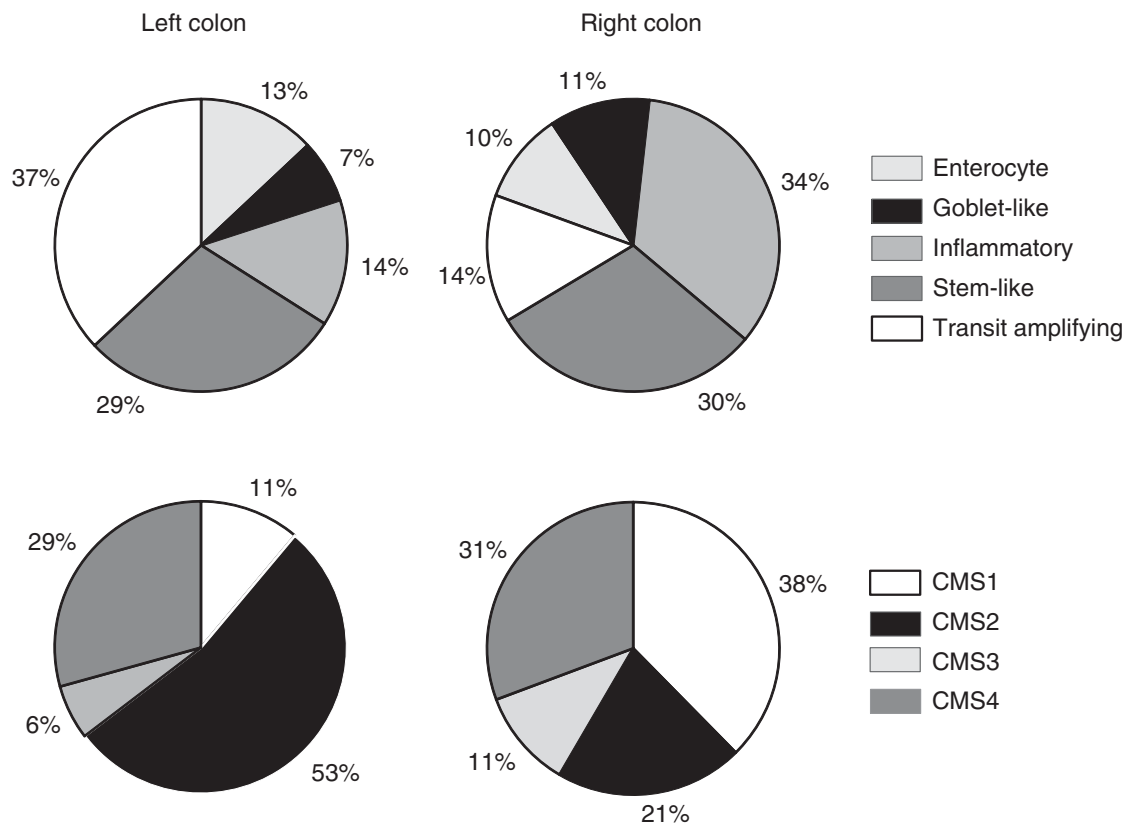

Figure 1. Distribution of intrinsic subtype frequency in left- and right-sided colon of all patients of NSABP/NRG C-07. CMS $=$ Consensus Molecular Subtype. 

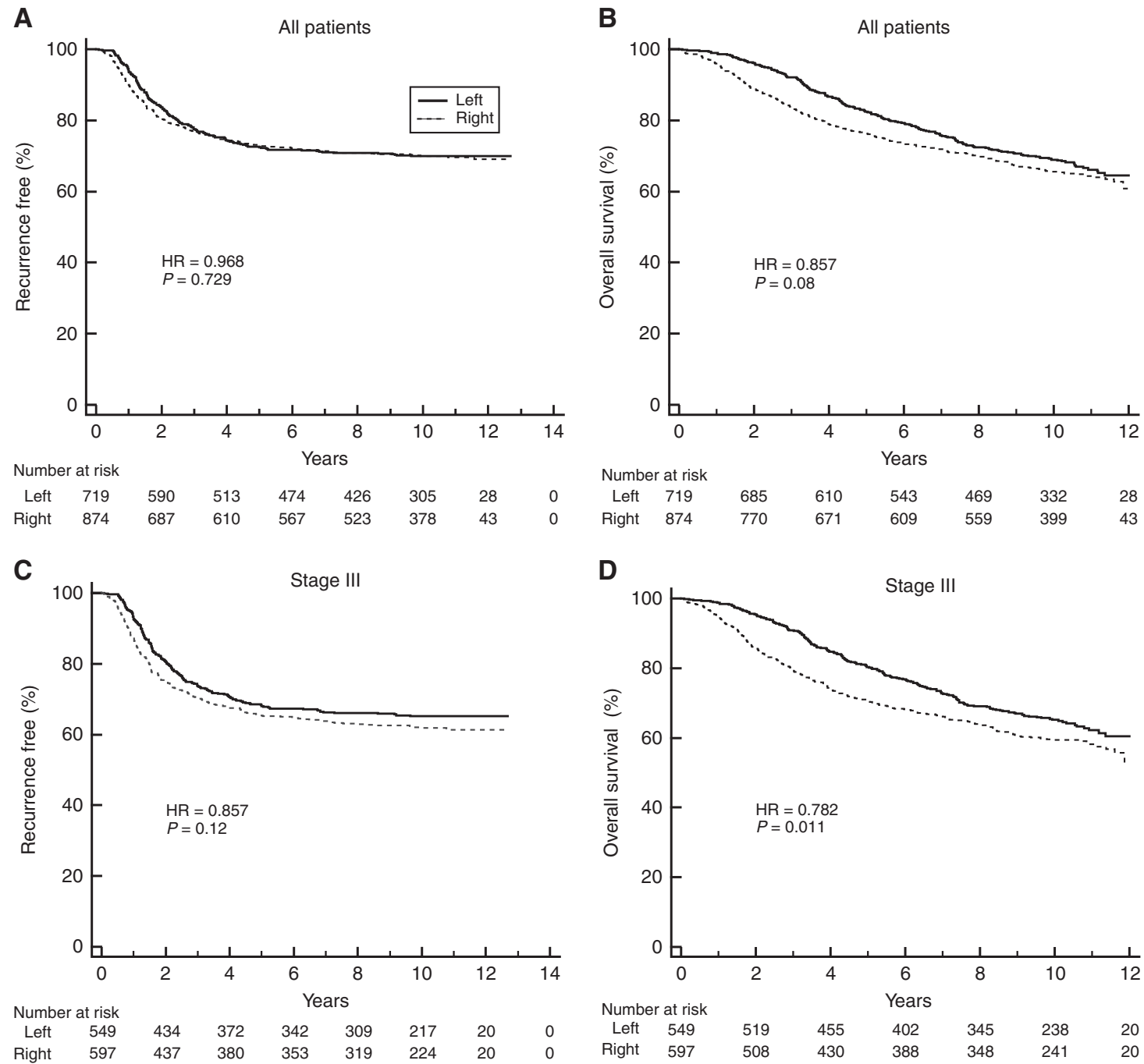

Figure 2. Kaplan-Meier plots for NSABP/NRG Oncology C-07 patients stratified by sidedness (left vs right) in: (A) all patients based on RFS, (B) all patients based on OS, (C) stage III patients based on RFS, and (D) stage III patients based on OS. The number of patients at risk is shown below the graph.

Figure 3B). We examined the association of sidedness with oxaliplatin benefit within the enterocyte subtype because this subset of patients received significant benefit from oxaliplatin (Song et al 2016). The oxaliplatin benefit based on RFS was greater in patients with stage III, left-sided tumours with an enterocyte subtype $(\mathrm{HR}=0.122(\mathrm{CI}: 0.036-0.411), P<0.001)$ than on rightsided tumours $(\mathrm{HR}=0.672$ (CI: $0.311-1.452), P=0.312)$, with a significant sidedness-oxaliplatin interaction based on RFS (interaction HR, $0.17 ; P=0.01$ ) (Figure $3 \mathrm{C}$ and $\mathrm{D}$ ) but not $\mathrm{OS}$ (interaction $\mathrm{HR}$ 0.51, $P=0.24$ ) (Figure $3 \mathrm{E}$ and $\mathrm{F}$ ). No difference between right- and left-sided tumours regarding benefit from oxaliplatin was seen in the 'other' CRCA subtypes composed of inflammatory, goblet-like, stem-like, and TA subtypes either based on RFS or OS (Figure 3D and F).

\section{DISCUSSION}

We saw an association of sidedness with prognosis in stage III C-07 patients based on OS but not in the entire cohort and not in stage III patients based on RFS. Patients in C-07 with left-sided tumours had superior OS. These results were similar to previous studies (Kerr et al, 2016; Schrag et al, 2016; Tejpar et al, 2016). Kerr et al
(2016) also found an association with sidedness and prognosis in stage II/III patients based on OS but not with RFS.

Right- and left-sided colon tumours both failed to show benefit from oxaliplatin in the $\mathrm{C}-07$ cohort in both the entire cohort and in stage III patients. Because our previously published results showed that only stage III patients with an enterocyte subtype tended to receive oxaliplatin benefit (Song et al, 2016), we examined oxaliplatin benefit in patients with enterocyte tumours and found that only patients with left-sided tumours received benefit from oxaliplatin; this remained significant even after adjusting for other clinical and molecular variables, including tumour sidedness $(P=0.005)$. No benefit from oxaliplatin could be detected in patients with right-sided enterocyte tumours. This analysis consisted of a small number of patients and these results should be interpreted cautiously.

With the exception of the poorest prognostic subtype (stemlike/CMS4), the distribution of molecular subtypes was different in the right and left side of the colon. This finding is consistent with those of several other publications, which may not be surprising because the right and left side of colorectal tumours arise from two different embryonic origins. Similar to the results seen in the metastatic setting, we also observed less treatment benefit in a small subset of C-07 patients with enterocyte, right-sided tumours (Tejpar et al, 2016). Other investigators have concluded that right- 
sided tumours may be more resistant to treatment, demonstrating that the overexpression of genes involved in cell cycle control in left-sided tumours might explain why these tumours are more

A

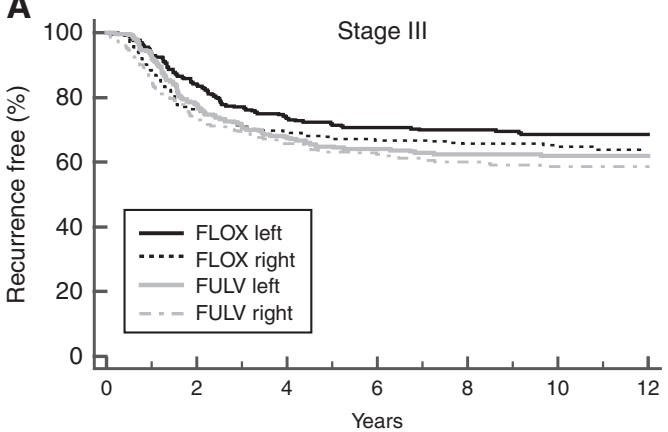

Number at risk FLOX lef

$\begin{array}{ccccccc}\begin{array}{c}\text { FLOX left } \\ 268\end{array} & 221 & 189 & 175 & 156 & 112 & 12 \\ \begin{array}{c}\text { FLOX right } \\ 312\end{array} & 234 & 202 & 189 & 177 & 130 & 10 \\ \begin{array}{c}\text { FULV left } \\ 281\end{array} & 213 & 183 & 167 & 153 & 105 & 8 \\ \begin{array}{c}\text { FULV right } \\ 285\end{array} & 203 & 178 & 164 & 142 & 94 & 10\end{array}$

C

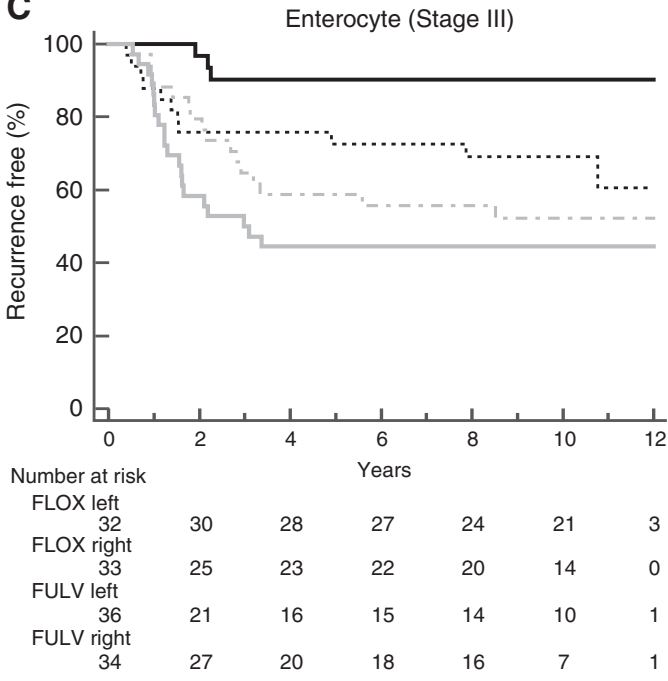

E

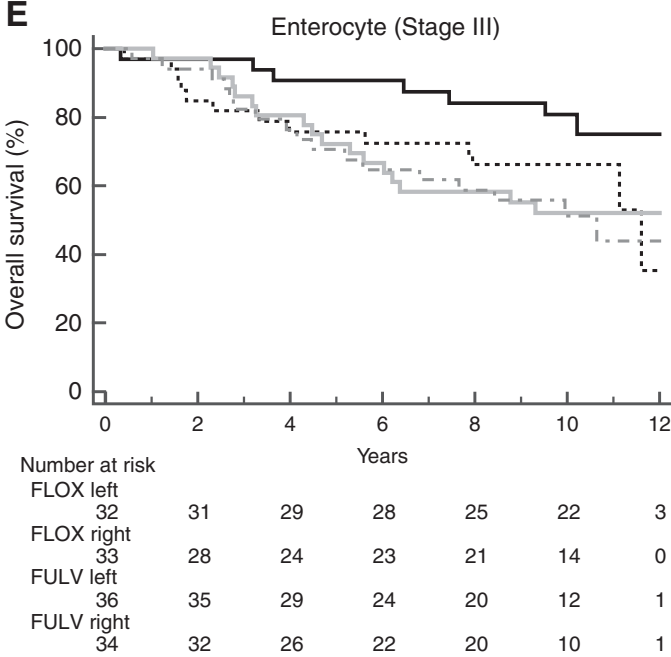

sensitive to cytotoxic therapies, but other pathways such as DNA damage repair, which are also overexpressed in the left, would suggest chemotherapy resistance (Glebov et al, 2003). The

B

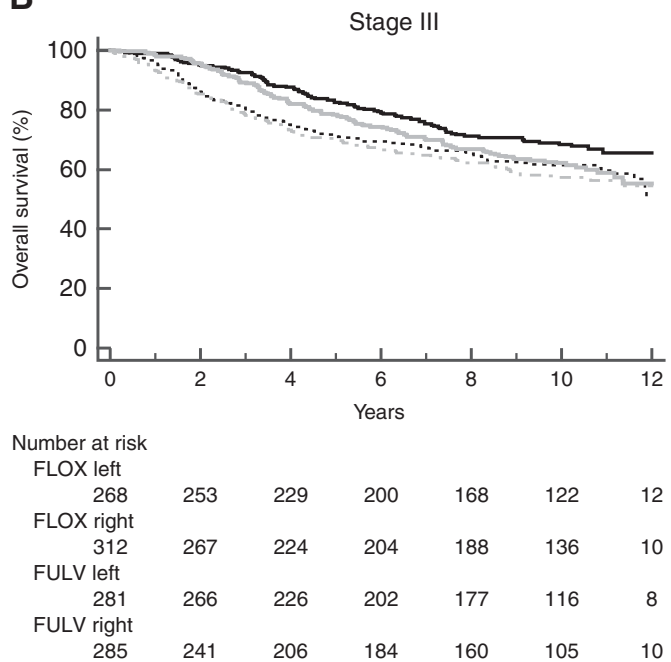

D

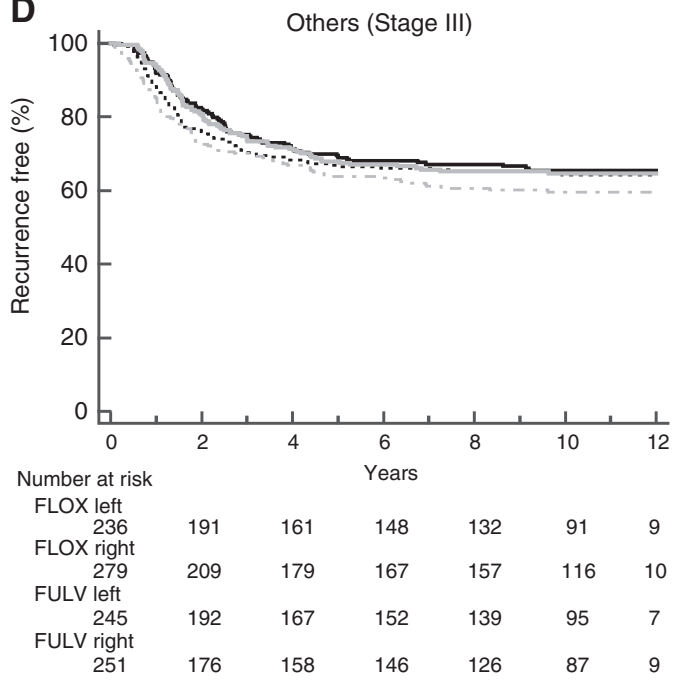

$\mathbf{F}$

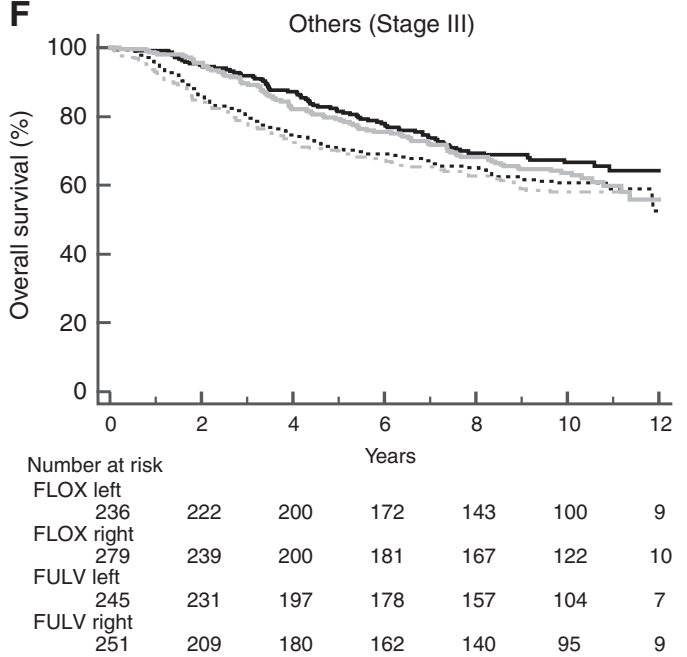

Figure 3. Kaplan-Meier plots for NSABP/NRG Oncology C-07 patients stratified by sidedness (left vs right) and treatment (5-fluorouracil (5FU) plus leucovorin (LV) vs 5-FU+LV and oxaliplatin (FLOX)) in: (A) stage III patients based on RFS, (B) stage III patients based on OS, (C) stage III enterocyte-subtype patients based on RFS, (D) stage III other-subtype patients based on RFS, (E) stage III enterocyte-subtype patients based on OS, and (F) stage III other-subtype patients based on OS. The number of patients at risk is shown below the graph. 
microbiome may be having a role in the differential response to therapeutic agents because biofilms that are more common on right-sided tumours are resistant to innate and adaptive immune responses (Bjarnsholt et al, 2013; Mima et al, 2016) and could act as a barrier to drug entry as well (Dejea et al, 2014).

The observation that stem-like/CMS4 (mesenchymal) subtype was evenly distributed between right and left colon in patients with stage III disease may have important clinical ramifications because these tumours are associated with a very poor prognosis and received no detectable benefit from oxaliplatin (Song et al, 2016). Thus clinical trials selecting patients with a poor prognosis should consider stratifying them according to molecular subtypes beyond tumour sidedness.

\section{ACKNOWLEDGEMENTS}

This work was supported by the National Cancer Institute at the National Institutes of Health, US Department of Health and Human Services, Public Health Service grants U10-CA180868, U10-CA180822, and U24-CA196067; Korea Health Technology R\&D Project through the Korean Health Industry Development Institute (KHIDI), funded by the Ministry of Health and Welfare, Republic of Korea (grant number HI13C2162) awarded to SP; a grant from the Pennsylvania Department of Health (The Department specifically disclaims responsibility for any analysis, interpretations, or conclusions.); and Sanofi-Synthelabo Inc. We thank Melanie Finnigan for data and tissue block management; William Hiller (deceased) and Teresa Oeler for histology; Wendy L Rea, Christine I Rudock, and Barbara C Good, PhD, for manuscript editing and preparation; Teresa $\mathrm{A}$ Bradley, $\mathrm{PhD}$, and Ethan Barry for regulatory affairs related to the manuscript; Francine Fonzi for protocol development; NSABP members who contributed tissue blocks; as well as patients who enrolled in the study. Funders had no role in study design, data collection or analysis, decision to publish, or preparation of the manuscript. SRK, KLP-G, and NS had full access to all the data in the study and take responsibility for the integrity of the data and the accuracy of the data analysis.

\section{CONFLICT OF INTEREST}

GY declares compensation by Pharmacyclics and Orbus Pharmaceutical companies for serving on data monitoring committees. The other authors declare no conflict of interest.

\section{REFERENCES}

Bjarnsholt T, Ciofu O, Molin S, Givskov M, Høiby N (2013) Applying insights from biofilm biology to drug development - can a new approach be developed? Nat Rev Drug Discov 12: 791-808.

Dejea CM, Wick EC, Hechenbleikner EM, White JR, Mark Welch JL, Rossetti BJ, Peterson SN, Snesrud EC, Borisy GG, Lazarev M, Stein E, Vadivelu J, Roslani AC, Malik AA, Wanyiri JW, Goh KL, Thevambiga I, Fu K, Wan F, Llosa N, Housseau F, Romans K, Wu X, McAllister FM, Wu S, Vogelstein B, Kinzler KW, Pardoll DM, Sears CL (2014) Microbiota organization is a distinct feature of proximal colorectal cancers. Proc Natl Acad Sci USA 111: 18321-18326.

De Sousa E Melo F, Wang X, Jansen M, Fessler E, Trinh A, de Rooij LP, de Jong JH, de Boer OJ, van Leersum R, Bijlsma MF, Rodermond $\mathrm{H}$, van der Heijden M, van Noesel CJ, Tuynman JB, Dekker E, Markowetz F, Medema JP, Vermeulen L (2013) Poor-prognosis colon cancer is defined by a molecularly distinct subtype and develops from serrated precursor lesions. Nat Med 19: 614-618.

Gavin PG, Colangelo LH, Fumagalli D, Tanaka N, Remillard MY, Yothers G, Kim C, Taniyama Y, Kim SI, Choi HJ, Blackmon NL, Lipchik C, Petrelli NJ, O'Connell MJ, Wolmark N, Paik S, Pogue-Geile KL (2012) Mutation profiling and microsatellite instability in stage II and III colon cancer: an assessment of their prognostic and oxaliplatin predictive value. Clin Cancer Res 18: 6531-6541.

Guinney J, Dienstmann R, Wang X, de Reyniès A, Schlicker A, Soneson C, Marisa L, Roepman P, Nyamundanda G, Angelino P, Bot BM, Morris JS, Simon IM, Gerster S, Fessler E, De Sousa E Melo F, Missiaglia E, Ramay H, Barras D, Homicsko K, Maru D, Manyam GC, Broom B, Boige V, Perez-Villamil B, Laderas T, Salazar R, Gray JW, Hanahan D, Tabernero J, Bernards R, Friend SH, Laurent-Puig P, Medema JP, Sadanandam A, Wessels L, Delorenzi M, Kopetz S, Vermeulen L, Tejpar S (2015) The consensus molecular subtypes of colorectal cancer. Nat Med 21: $1350-1356$.

Glebov OK, Rodriguez LM, Nakahara K, Jenkins J, Cliatt J, Humbyrd CJ, DeNobile J, Soballe P, Simon R, Wright G, Lynch P, Patterson S, Lynch H, Gallinger S, Buchbinder A, Gordon G, Hawk E, Kirsch IR (2003) Distinguishing right from left colon by the pattern of gene expression. Cancer Epidemiol Biomarkers Prev 12: 755-762.

Kerr DJ, Domingo E, Kerr R (2016) Is sidedness prognostically important across all stages of colorectal cancer? Lancet Oncol 17: 1480-1482.

Mima K, Cao Y, Chan AT, Qian ZR, Nowak JA, Masugi Y, Shi Y, Song M, da Silva A, Gu M, Li W, Hamada T, Kosumi K, Hanyuda A, Liu L, Kostic AD, Giannakis M, Bullman S, Brennan CA, Milner DA, Baba H, Garraway LA, Meyerhardt JA, Garrett WS, Huttenhower C, Meyerson M, Giovannucci EL, Fuchs CS, Nishihara R, Ogino S (2016) Fusobacterium nucleatum in colorectal carcinoma tissue according to tumor location. Clin Transl Gastroenterol 7: e200.

Sadanandam A, Lyssiotis CA, Homicsko K, Collisson EA, Gibb WJ, Wullschleger S, Ostos LC, Lannon WA, Grotzinger C, Del Rio M, Lhermitte B, Olshen AB, Wiedenmann B, Cantley LC, Gray JW, Hanahan D (2013) A colorectal cancer classification system that associates cellular phenotype and responses to therapy. Nat Med 19: 619-625.

Schrag D, Weng S, Brooks G, Meyerhardt JA, Vennok AP (2016) The relationship between primary tumor sidedness and prognosis in colorectal cancer. ASCO Meeting Abstracts; June 3-7, 2016; Chicago, IL. J Clin Oncol 34(Suppl): Abstr 3505.

Song N, Pogue-Geile KL, Gavin PG, Yothers G, Kim SR, Johnson NL, Lipchik C, Allegra CJ, Petrelli NJ, O'Connell MJ, Wolmark N, Paik S (2016) Clinical outcome from oxaliplatin treatment in stage II/III colon cancer according to intrinsic subtypes: secondary analysis of NSABP C-07/ NRG Oncology randomized clinical trial. JAMA Oncol 2: 1162-1169.

Tejpar S, Stintzing S, Ciardiello F, Tabernero J, Van Cutsem E, Beier F, Esser R, Lenz HJ, Heinemann V (2016) Prognostic and predictive relevance of primary tumor location in patients with RAS wild-type metastatic colorectal cancer: retrospective analyses of the CRYSTAL and FIRE-3 Trials (2016). JAMA Oncol 3(2): 194-201.

Venook AP, Niedzwiecki D, Innocenti F, Fruth B, Greene C, O’Neil BH, Shaw JE, Atkins JN, Horvath LR, Polite BN, Meyerhardt JA, O’Reilly EM, Goldberg RM, Hochster HS, Blanke CD, Schilsky RL, Mayer RJ, Bertagnolli MM, Lenz H-J (2016) Impact of primary tumor location on overall survival and progression-free survival in patients with metastatic colorectal cancer: analysis of CALGB/SWOG 80405 (Alliance). ASCO Meeting Abstracts; June 3-7; Chicago, IL, J Clin Oncol, Abstr 3504.

This work is published under the standard license to publish agreement. After 12 months the work will become freely available and the license terms will switch to a Creative Commons AttributionNonCommercial-Share Alike 4.0 Unported License.

Supplementary Information accompanies this paper on British Journal of Cancer website (http://www.nature.com/bjc) 